\title{
Design, manufacturing and measurements of a metal-less V-groove RXI collimator
}

\author{
Dejan Grabovičkić a , Juan C. Miñano ${ }^{\text {ab }}$, Pablo Benítez ${ }^{\text {ab }}$, Cristina Cocho ${ }^{\text {a }}$, Marina Buljan ${ }^{\text {a }}$ \\ ${ }^{a}$ CEDINT, Universidad Politécnica de Madrid (UPM), Campus de Montegancedo 28223 Pozuelo de \\ Alarcón, Madrid, Spain \\ ${ }^{\mathrm{b}}$ Light Prescriptions Innovators. 16662 Hale Ave., Irvine, CA USA 92606
}

\begin{abstract}
A metal-less RXI collimator has been designed. Unlike to the conventional RXI collimators, whose back surface and central part of the front surface have to be metalized, this collimator does not include any mirrored surface. The back surface is designed as a grooved surface providing two TIR reflections for all rays impinging on it. The main advantage of the presented design is lower manufacturing cost since there is no need for the expensive process of metalization. Also, unlike to the conventional RXI collimators this design performs good colour mixing. The first prototype of Vgroove RXI collimator has been made of PMMA by direct cutting using a five axis diamond turning machine. The experimental measurements of the first prototype are presented.
\end{abstract}

Keywords: geometrical optics, optical design, RXI collimator, V-grooves, LED, nonimaging optics

\section{INTRODUCTION}

A RXI collimator consists of two surfaces: front surface and back surface, Figure 1. The name of the collimator comes from the description of the ray trajectories. A ray coming from the LED is reflected first by TIR at the front surface, then is reflected specularly at the mirrored back surface and finally leaves the collimator being refracted at the front surface. There is a small central region at the front surface that has to be metalized as well, since the TIR conditions are not satisfied for the rays impinging on this surface for the first time [1].

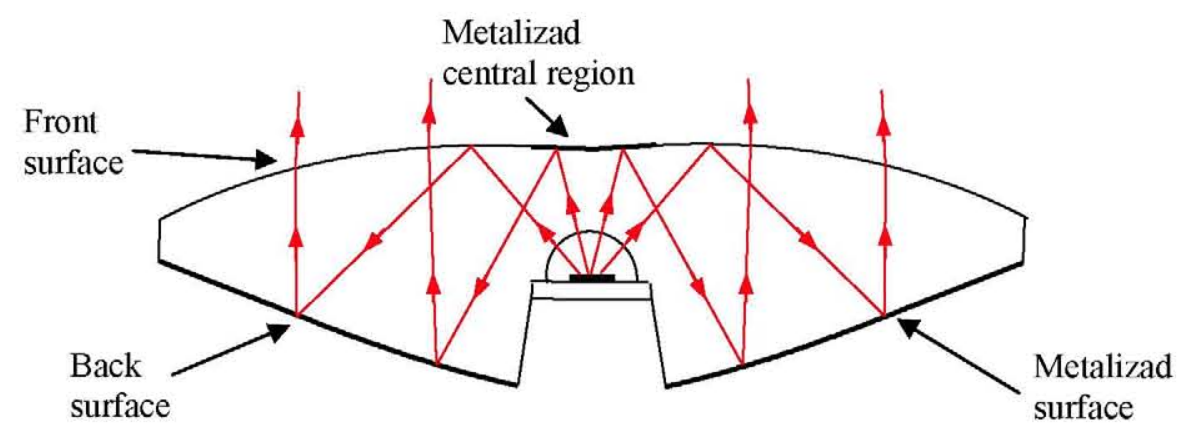

Figure 1. RXI collimator.

The LED and the collimator must be joined with silicon to avoid influence of the air gap. This complicates the manufacture of the design, so the next architecture was proposed by Muñoz and Benítez [2], [3], Figure 2. This collimator contains an additional surface that separates the LED from the optics. Now, along the ray path there is another refraction (at the entrance surface), so this collimator is called RIXR. The back surface and the central part of the front surface are metalized like in the previous design. 


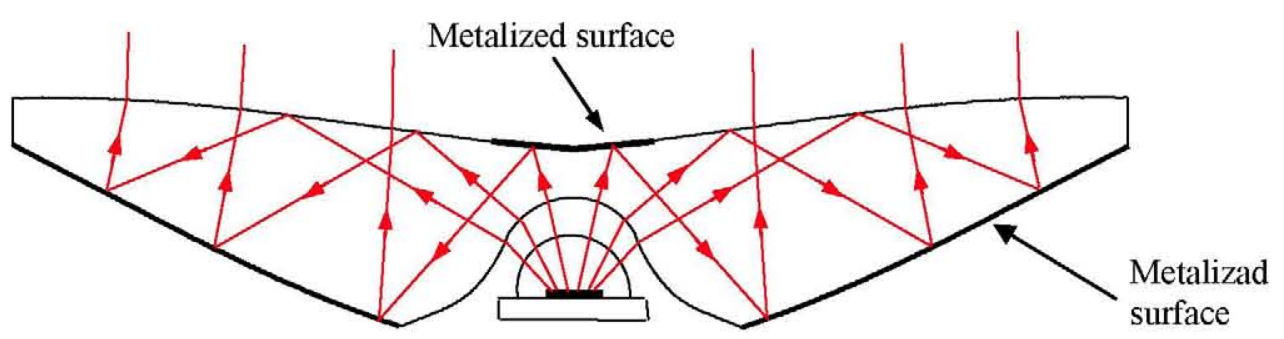

Figure 2. RIXR collimator.

\section{V-GROOVE RXI COLLIMATOR}

Herein, we present a metal-less V-groove RXI collimator. A ray coming from a light source refracts first at the entrance surface, then reflects at the front surface by TIR, and reaches the back surface. There, on the back surface the ray is reflected twice by TIR at each groove side (Figure 3) and redirected toward the front surface, where the ray will be refracted again. Considering the ray path from the source (in this case a LED), each ray suffers five deflections: a refraction, three total internal reflections, and again refraction. Thus, this design can be named also $\mathrm{RI}^{3} \mathrm{R}$ collimator.

(a)

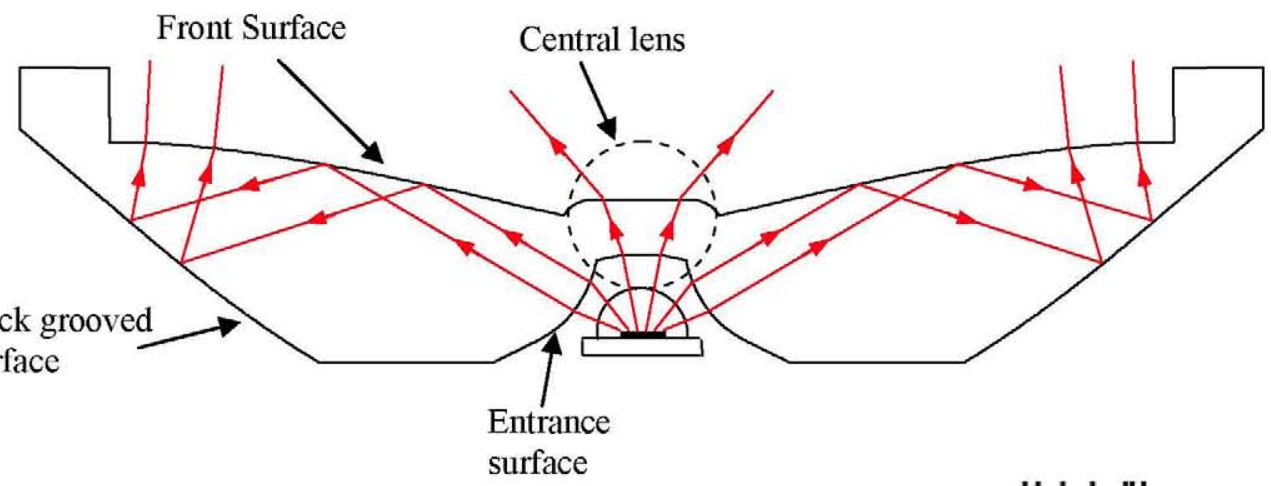

(b)

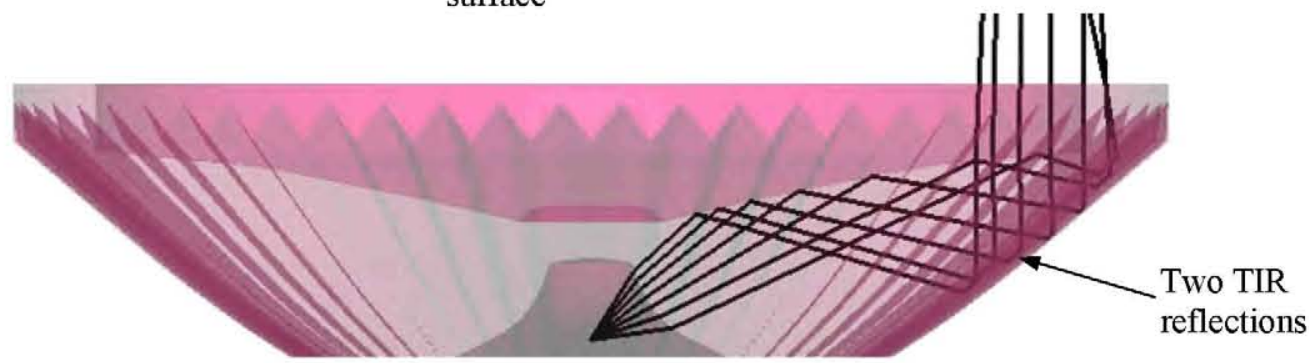

Figure 3. Metal-less V-groove RXI. (a) Cros section (b) 3D View with the rays traced in LightTools.

The central part of the front surface can be replaced by a grooved surface as well. However, since this surface is close to the optical axis, the grooves are very small. This complicates the fabrication process, so we decided to replace the central part by a lens. This lens provides smooth intensity pattern for higher angles (from $10^{\circ}-30^{\circ}$ ) as shown in Figure (Section 3.3)

\section{DESIGN PROCEDURE}

\subsection{D design}

The first step in calculation of the V-groove RXI is 2D RIXR design, which is also explained in [2]. In order to design the collimator we need to couple the edge rays of the input and the output bundle (Figure 4). The LED is considered as a perfect Lambertian source, thus the set of the edge rays of the input bundle is defined completely by the rays coming 
from the extreme LED points $\mathrm{L}_{-}$and $\mathrm{L}_{+}$. The edge rays of the output bundle consist of the rays that arise from the strip $\mathrm{P}_{0} \mathrm{Q}_{0}$ (also the strip can have a dark zone in the centre, defined by the parameter $\mathrm{x}_{\mathrm{D}}$ ) and make the angle $\alpha$ with the optical axis $z$, and also the rays coming from the extreme points $\mathrm{P}_{0}$ and $\mathrm{Q}_{0}$ forming an angle smaller or equal to $\alpha$ (Figure 4).

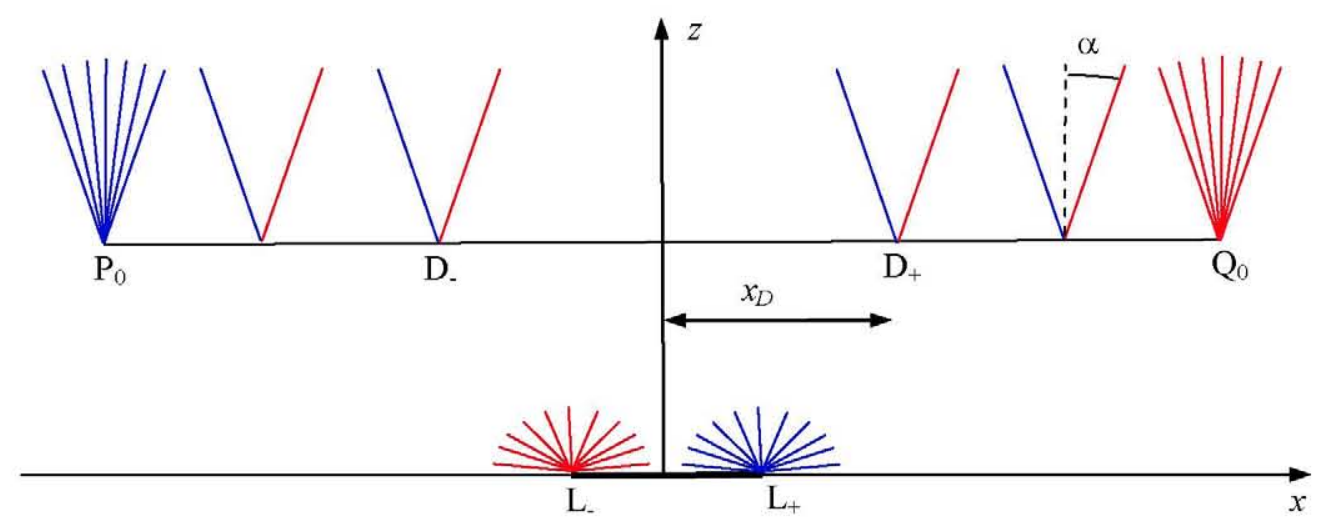

Figure 4. Definition of the edge rays for input and output bundles.

Since the RIXR is symmetric respect to the optical axis $z$, we will explain design procedure only for a half of the collimator.

The design procedure starts by defining the first segment of the front surface $\mathrm{A}_{0} \mathrm{~A}_{2}$ (Figure 5). This segment is taken as an arc of circumference. Consider the edge rays at an extreme point of the strip $\mathrm{P}_{0} \mathrm{Q}_{0}$. These rays are contained between the rays $q_{0 \text { - }}$ and $q_{0^{+}}$, as shown in Figure 5. First, we refract them at the front surface, and then we calculate the first segment of the back surface $\mathrm{B}_{0} \mathrm{~B}_{1}$ as a Cartesian Oval surface providing that all these rays are reflected toward the point $\mathrm{A}_{2}$. Next, we need to check the TIR conditions at the point $\mathrm{A}_{2}$. If they are not satisfied, we back to the beginning of the procedure and prescribe a different form of the segment $\mathrm{A}_{0} \mathrm{~A}_{2}$. Afterwards, the first segment of the entrance surface $\mathrm{C}_{0} \mathrm{C}_{1}$ is calculated as a Cartesian Oval which couples the reflected rays at $\mathrm{A}_{2}$ and the input edge rays coming from $\mathrm{L}_{-}$, after the refraction at $\mathrm{C}_{0} \mathrm{C}_{1}$. Once $\mathrm{C}_{0} \mathrm{C}_{1}$ is calculated, the entire entrance surface is defined as an arc of circumference $\mathrm{C}_{1} \mathrm{C}_{n}$ (the dashed arc in Figure 5), having the same derivative as the segment $\mathrm{C}_{0} \mathrm{C}_{1}$ at $\mathrm{C}_{1}$.

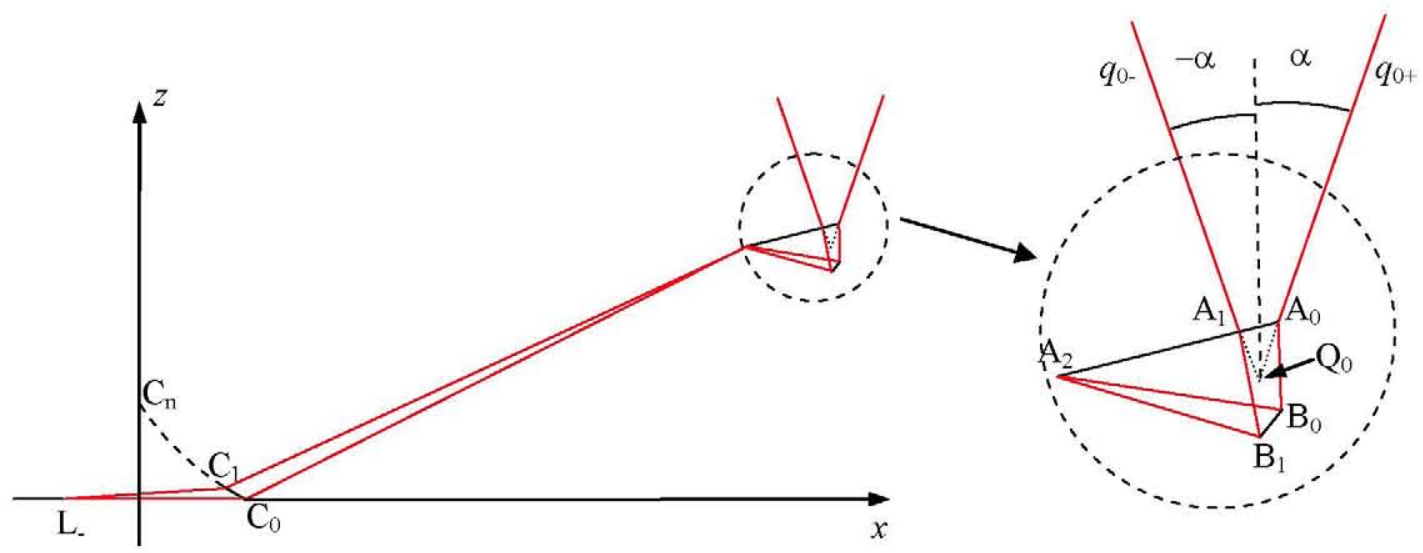

Figure 5. Calculation of the first segments of the back and entrance surface.

Next, we use the calculated piece of the back surface $\mathrm{B}_{0} \mathrm{~B}_{1}$ to find more points of the front surface. Let refract now the set of parallel rays, contained between $q_{0+}$ and $q_{x^{+}}$(Figure 6), at the front surface, then reflect them at the previously calculated segment $\mathrm{B}_{0} \mathrm{~B}_{1}$. Since the entrance surface is completely defined, we are able to find the new piece of the front surface $\mathrm{A}_{2} \mathrm{~A}_{3}$ as Cartesian Oval that couples the rays coming from $\mathrm{B}_{0} \mathrm{~B}_{1}$ and $\mathrm{L}_{-}$(Figure 6). 


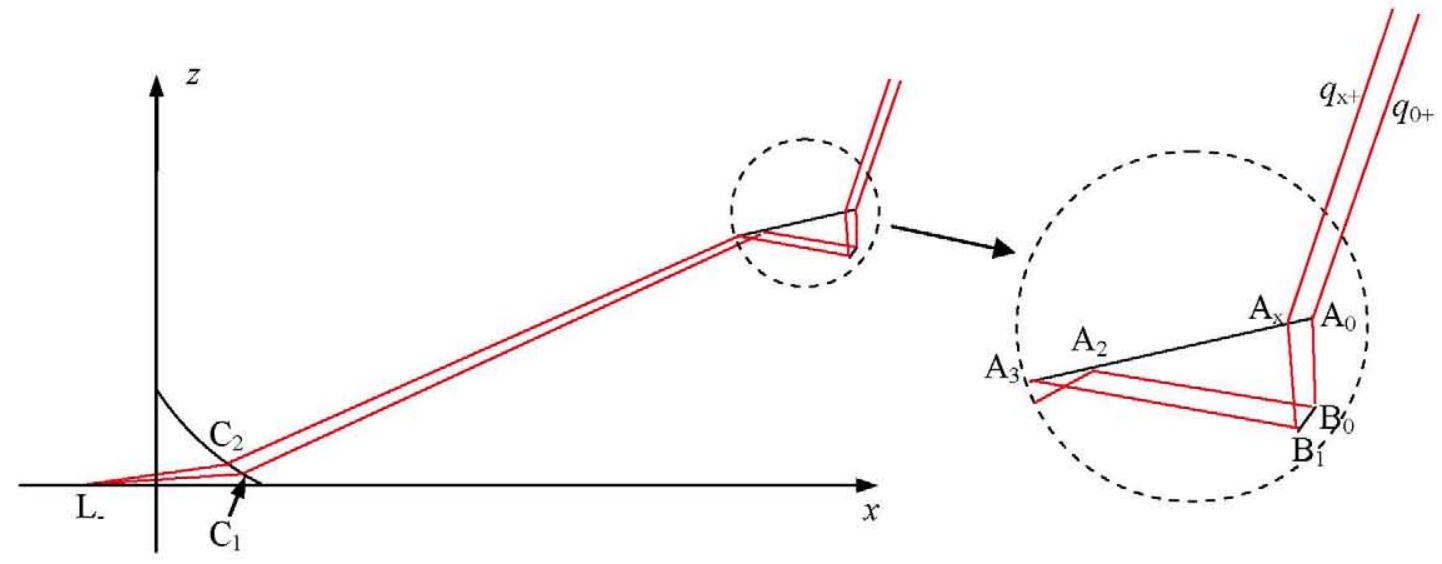

Figure 6. Calculation of the second segment of the front surface $\mathrm{A}_{2} \mathrm{~A}_{3}$.

Now we trace the rays from another LED extreme point $\mathrm{L}_{+}$. These rays are reflected at the previously calculated segment $\mathrm{A}_{2} \mathrm{~A}_{3}$ and then coupled with the set of parallel rays contained between $q_{0-}$ and $q_{y .}$. As the result of this coupling, a new piece of the back surface $B_{1} B_{2}$ is obtained (Figure 7).

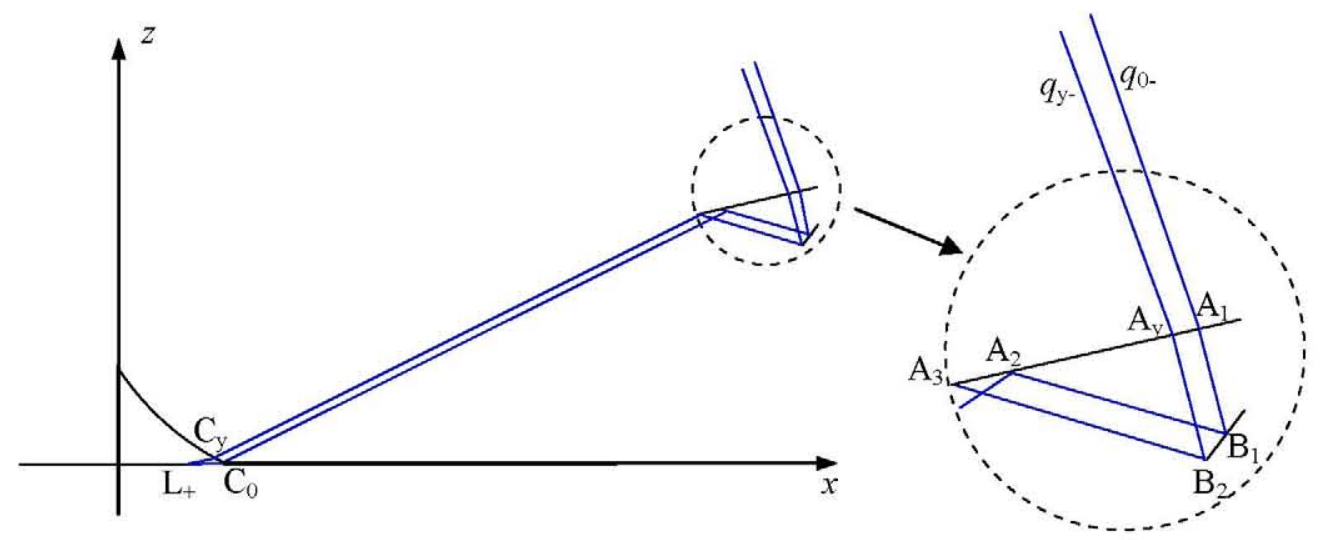

Figure 7. Calculation of the second segment of the back surface $\mathrm{B}_{1} \mathrm{~B}_{2}$.

The procedure continues thereafter repeating alternately the last two steps (shown in Figure 6 and Figure 7) to build entirely both surfaces. This procedure is called Simultaneous Multiple Design method in two dimensions (SMS2D) [4]. Each new segment of the back surface is obtained by coupling the rays from $\mathrm{L}_{+}$with the rays that leave the RIXR making the angle $-\alpha$ respect to $z$. The calculation of the front surface progresses using the rays coming from $\mathrm{L}_{\text {- }}$ and the rays that leave RIXR forming the angle $\alpha$ respect to $z$.

Note, that during the procedure it has to be checked each reflection at the front surface. If the TIR conditions are not satisfied, the procedure stops.

\subsection{D V-groove RXI design}

Once the 2D model is designed, we replace the mirrored back surface by a properly calculated grooved reflector. Since, the entrance and the front surface are known, we are able to calculate the wavefronts coming to the mirrored surface and reflecting from it. Denote them as $W F_{1}$ and $W F_{2}$. Then, the collimator's back surface (Figure 8 ) can be constructed as a grooved surface using the procedure explained in [5]. The V-grooved surface provides coupling between two wavefronts after two TIR reflections at the V-groove, no matter which side of the groove the rays hit first. 


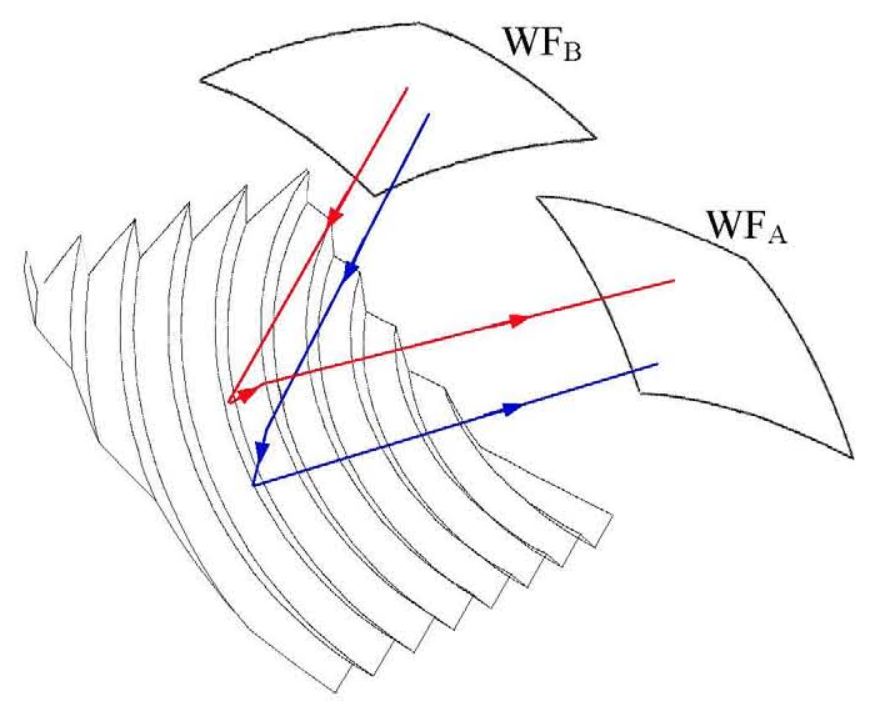

Figure 8. Grooved surface substitute for the RIXR back surface.

In general, each groove comprises two free-form surfaces joined along the groove edge-line (Figure 9.a)). However, for the sake of easier fabrication, we decided to approximate the design using the flat V-grooves. A flat groove has a freeform edge-line, but its cross section (the plane perpendicular to the edge-line) is merely a $90^{\circ}$ corner (the line in red, Figure 9.b)). Since the V-groove sides are mutually perpendicular at the groove edge-line [5], this approximation is very good as long as the grooves are small. In our case, the groove-edge line coincides with the curve that defines back surface in 2D RIXR model (which is not a straight line).

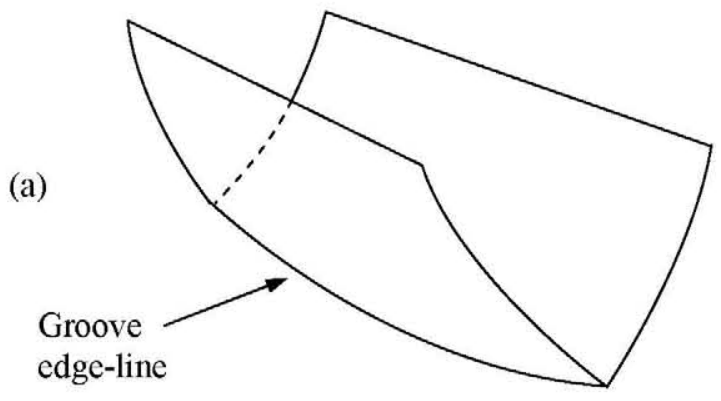

(b)

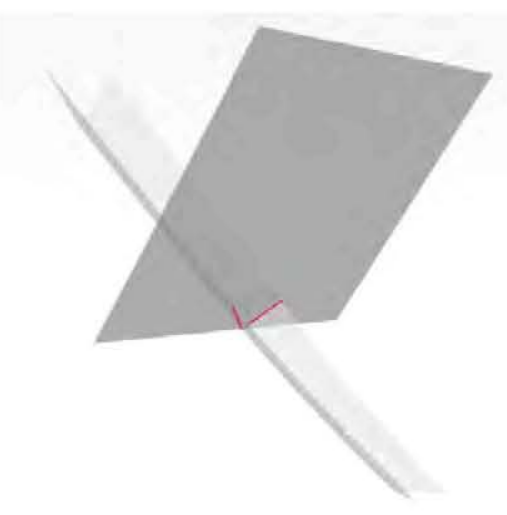

Figure 9. a) Free-form groove, b) Flat V-groove. The cross section is a $90^{\circ}$ corner (the line in red).

The number of the V-grooves in the presented design can be chosen freely. The larger the number is, the smaller the grooves are. Due to the restrictions on groove's size given by the manufacturer, we have designed model with 60 equal flat V-grooves (Figure 10). The size of the $90^{\circ}$ groove's cross section changes along the curved edge-line, from $c=0.6$ $\mathrm{mm}$ to $c=1.15 \mathrm{~mm}, c$ being the longitud of each corner side. Figure 10 shows the final V-groove RXI design, the one that has been chosen for the protype. This model has been fabricated and characterized. 


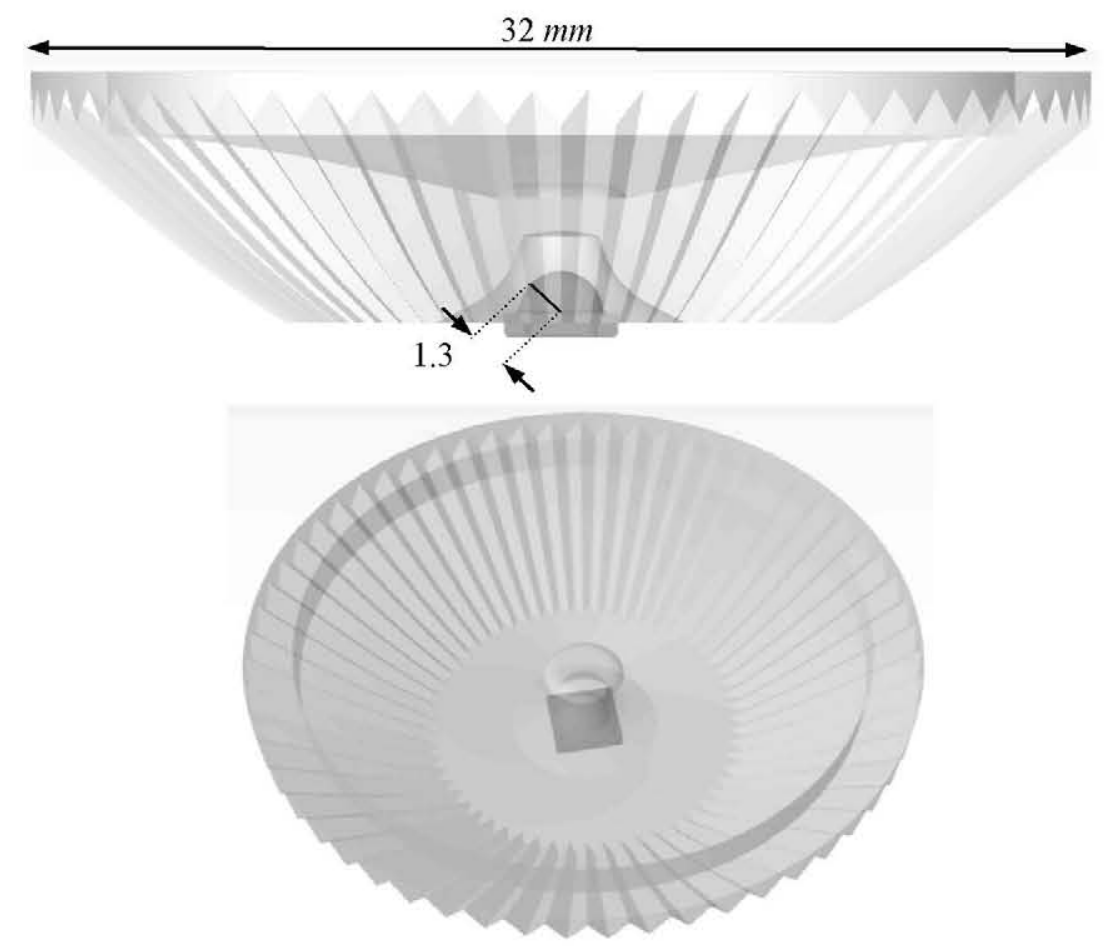

Figure 10. V-groove RXI model for fabrication with XP-G Cree LED.

\subsection{Simulations of the design}

The V-groove RXI prototype is simulated in LightTools [8]. The LED has been modeled using the ray set of XP-G Cree white LED (this ray set is provided by the manufacturer). Figure 11 shows simulated far field pattern (LED power is 100 lm).
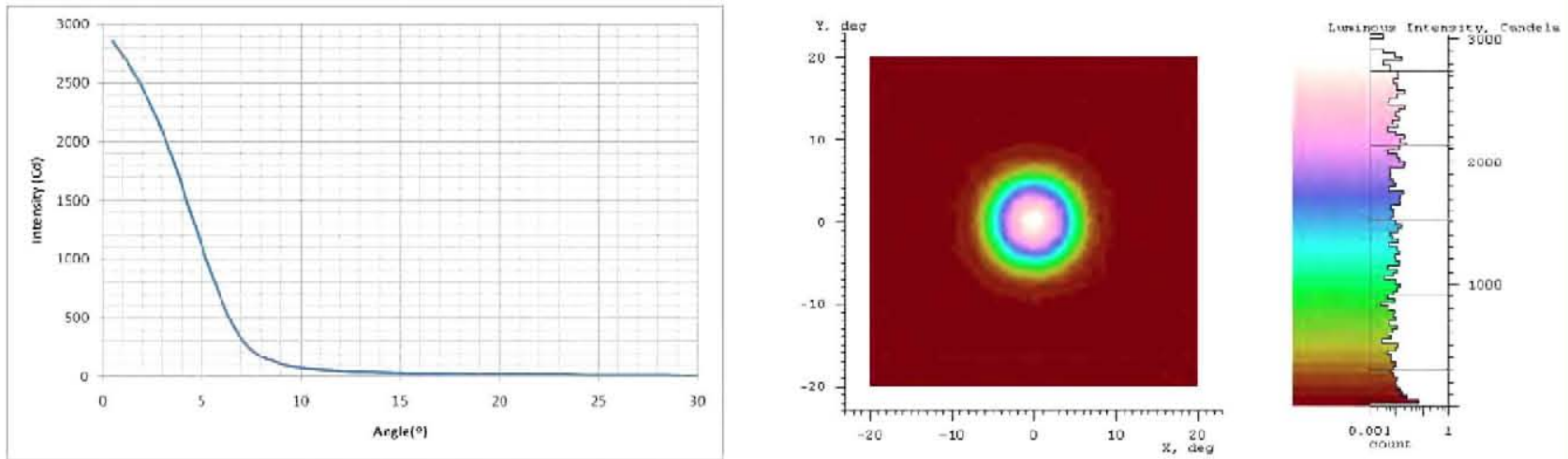

Figure 11. Far field pattern obtained in LightTools.

Most of the power have reached the far field receiver inside the square $10^{\circ} \times 10^{\circ}$ (about $69.1 \%$ ), about $88.6 \%$ of the power reaches inside the square $30^{\circ} \times 30^{\circ}$. Smooth part of the intensity pattern (from $10^{\circ}-30^{\circ}$ ) is obtained by a proper design of the central lens.

\section{COLOUR MIXING}

The V-groove RXI, unlike to the others metalized RXI mixes well the colours. This means that RGB LED chips can be used, as well. It is well known that the conventional RXI designs form good image of the source. This feature is bad when colour mixing is needed. When four different colour LED chips are used (for example RGGB or RGBW LED), 
since the far field pattern replicates well the source image, in the far field we will have four separated images of each chip. Colour mixing features of both designs are simulated in LightTools. The LEDs are modeled as perfect Lambertian sources emitting in different wavelengths. We put two green LEDs, one blue and one red LED (Figure 12). The spacial distribution of these LEDs, their size, and optical properties are similar to a conventional RGB LED.

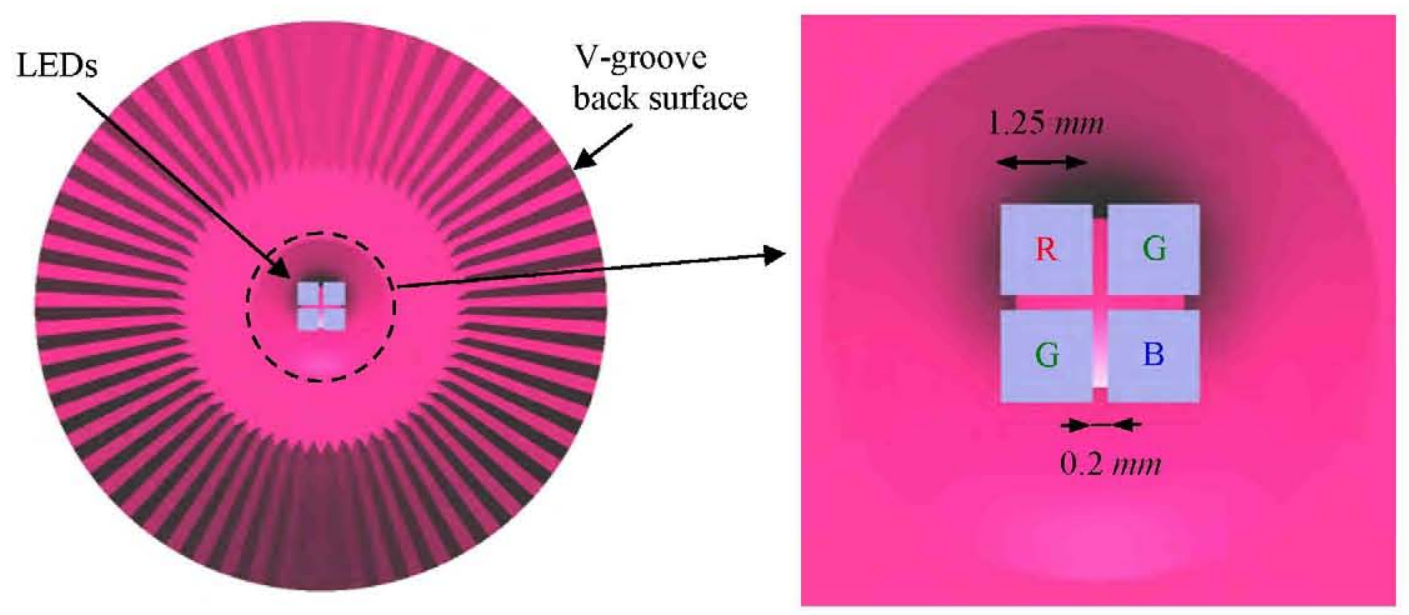

Figure 12. Set up for LightTools colour mixing simulation.

Figure 13.a) shows the simulated true colour far field pattern for metalized RIXR. Although the collimator do not form perfect image of the source (look the image edges), the colours are not mixed, and four images of each chip can be perceived. Figure 13.b) shows true colour far field for the metal-less RXI reflector. Instead of the source image in the far field, we have obtained a very good colour mixing. This design performs a rotation of the far field pattern. The far field pattern coming from each point source has ringlike shape. This effect is explained in [6], [7].

(a)

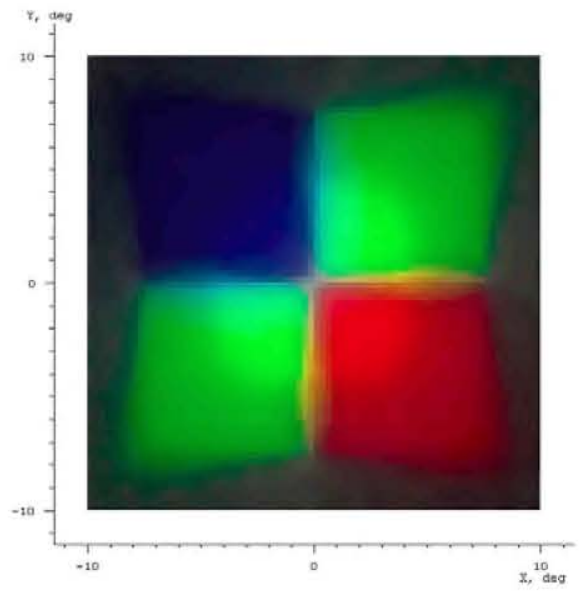

(b)

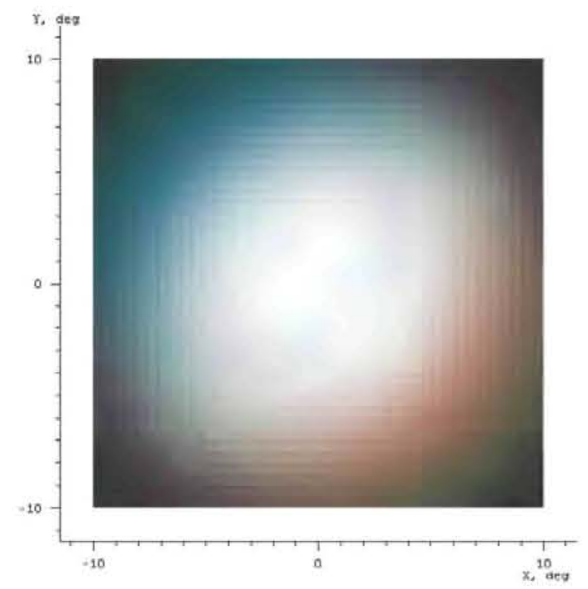

Figure 13. Colour mixing, real colour far field patterns a) RIXR b) metal-less V-groove RXI collimator.

We have done the same simulations using 4 white LEDs instead of the RGGB. We have put two warm white, one cool white and one neutral white LED (the power of each LED is different, without any optics they provide white colour). Again we can see that conventional RIXR provide a source image (Figure 14.a)) instead of colour mixing (some areas are darker, since the power of these LEDs are much lower). Our metal-less RXI again performs very good colour mixing (Figure 14.b)). 
(a)

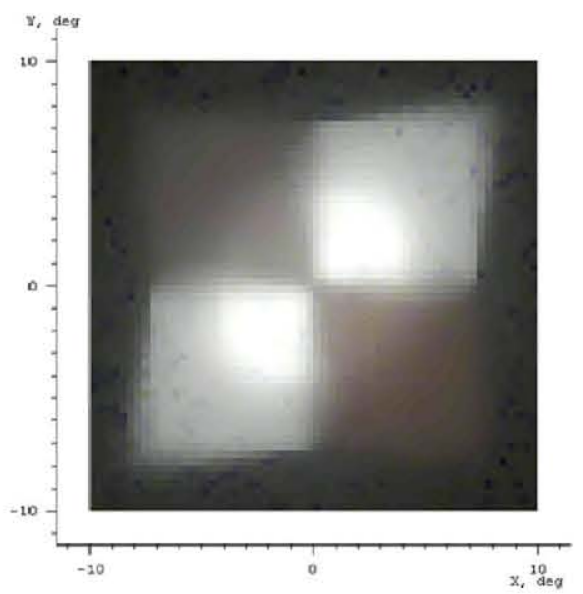

(b)

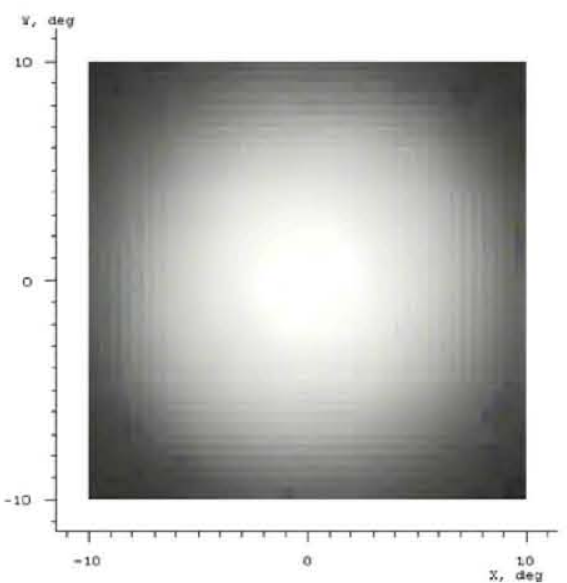

Figure 14. Colour mixing for 4 white LEDs. Real colour far field patterns a) RIXR b) metal-less V-groove RXI collimator.

\section{EXPERIMENTAL RESULTS OF THE FIRST PROTOYPE}

In order to analyze the potential of the V-groove RXI collimators a prototype was fabricated. Figure 15 shows the first V-groove RXI prototype, made of PMMA by direct cutting. The geometrical measurements of the prototype have proven good quality of cutting process. This means that the surface roughness is low and that the curvature radio of the groove peaks are small. The edges have been observed under a microscope and it has been noticed that the edge curvatures fluctuates being smaller than $10 \mu \mathrm{m}$, which is the tolerance of the manufacturer.
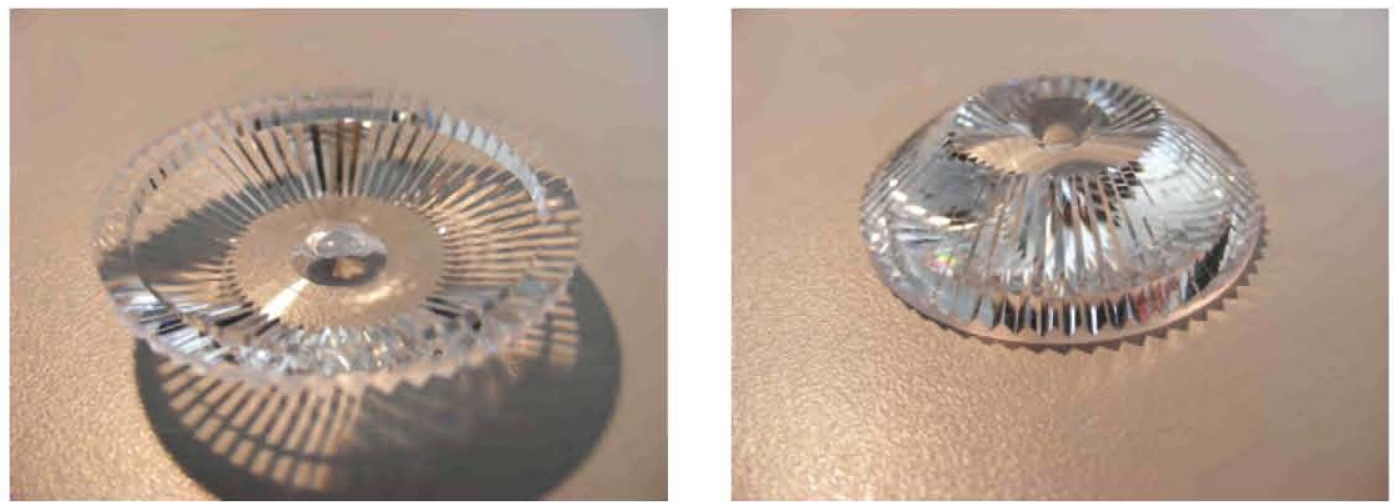

Figure 15. Metal-less V-groove RXI collimator. Fabricated prototype

For the measurement the V-groove RXI and a XP-G Cree LED are fixed on a supportive structure as shown in Figure 16.
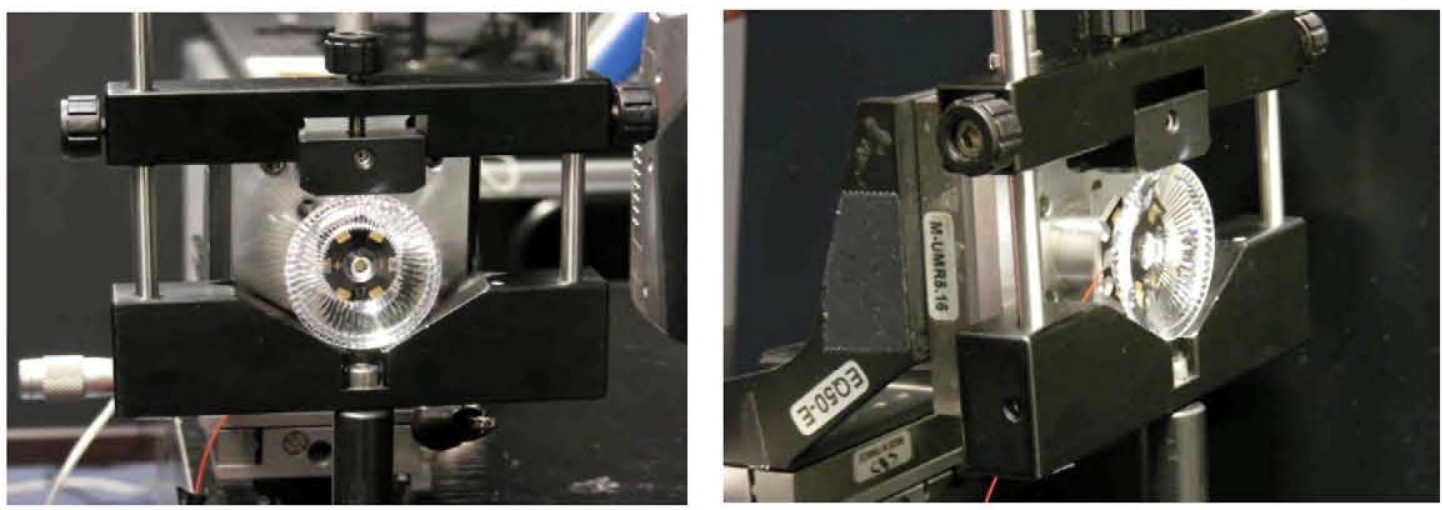

Figure 16. Fixture of the V-groove RXI collimator and the LED. 
Figure 17 shows front view of the set up. In the picture on the right side, the LED was switched off, while in the picture on the left it was switched on. The grooved surface is shiny, since the rays are reflected on it.

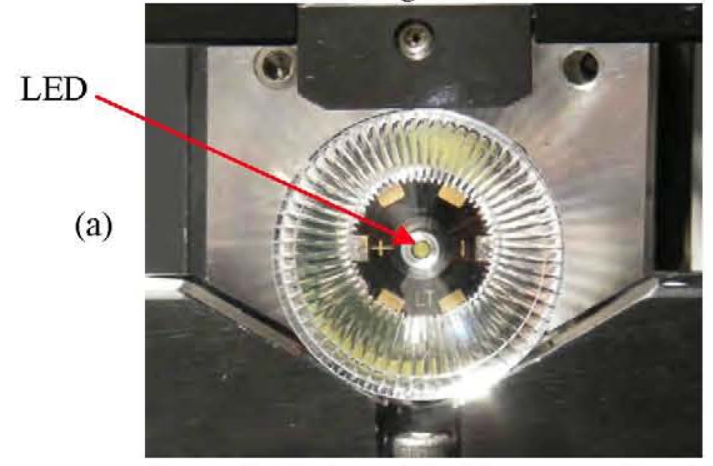

(b)

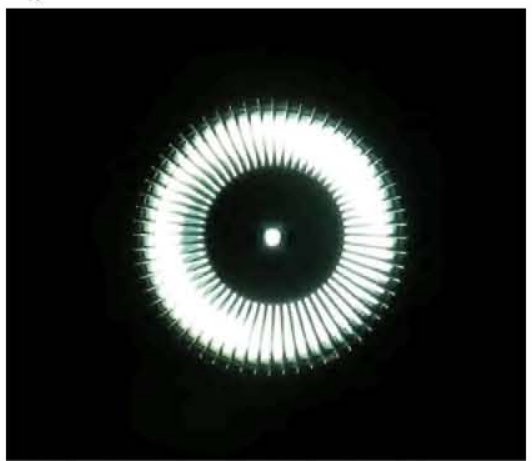

Figure 17. Fixture of the V-groove collimator and the LEd. a) The LED is off, b) The LED is on.

Figure 18 shows real far field pattern for the V-groove RXI. We have captured two far field images, first, the one of the V-groove RXI, and then, the one of the LED (without RXI optics). According to these measurements, $70.1 \%$ of the input power reaches the far field inside the rectangular $\pm 15^{\circ}$. This is very good result, since in the theoretical case (the LightTools simulation) it is $74.9 \%$

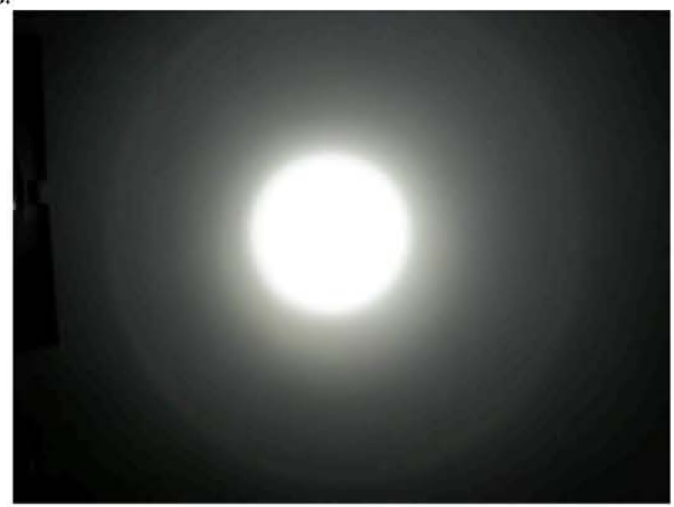

Figure 18. V-groove RXI prototype. Real photo of the far field pattern.

Figure 19 shows the measured and the simulated intensity distribution (for the input power of $1 \mathrm{~lm}$ ). As one can see, both graphs are very similar, with the same value of the full-width half-maximum angle of about $4.5^{\circ}$. The maximum value of the measured intensity $22 \mathrm{~cd} / / m$ is slightly lower than the simulated one $(28 \mathrm{~cd} / / m)$.

(a)

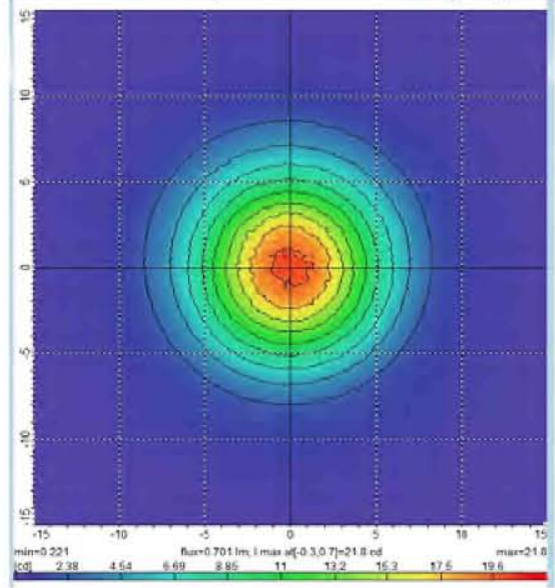

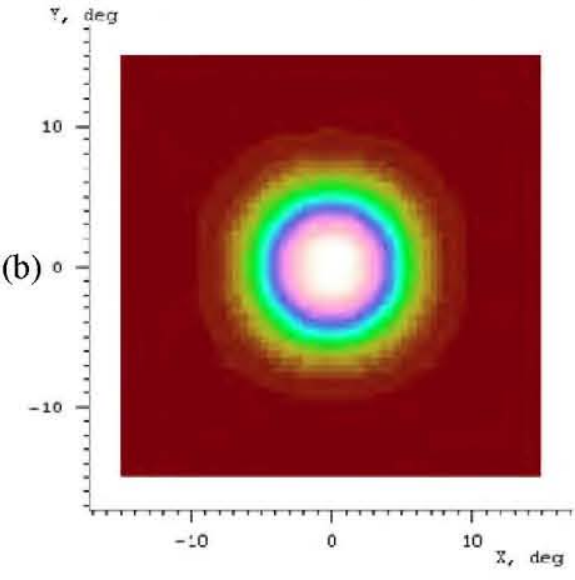

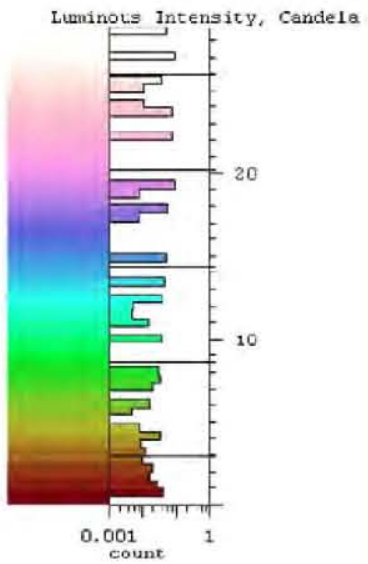

count

Figure 19. Intensity distribution in the far field (for the input power of $1 \mathrm{~lm}$ ). a) real measured distribution b) intensity distribution obtained in LightTools. 


\section{CONCLUSIONS}

A metal-less V-groove RXI design has been presented. One of its sides is a properly calculated grooved surface that reflects the rays by two TIR reflections, acting as a mirrored surface in the conventional RXI. A V-groove RXI design with 60 flat V-grooves is designed, fabricated, and characterized. The measurements of the fabricated piece show good efficiency $70.1 \%$, inside the rectangular $\pm 15^{\circ}$ (about $94 \%$ of the theoretical value). Also, the measured far field pattern coincides with the simulated one. The maximum value of the measured intensity is $22 \mathrm{~cd} / \mathrm{lum}$. Unlike to the conventional RXI collimators, V-groove RXI performs good colour mixing. Since the presented design does not need any metalization, the manufacturing cost is lower than in the case of conventional RXI.

\section{ACKNOWLEDGMENTS}

Authors thank the Spanish Ministries MCINN (ENGINEERING METAMATERIALS: CSD2008-00066, DEFFIO: TEC2008-03773, SIGMASOLES: PSS-440000-2009-30), MITYC (ECOLUX: TSI-020100-2010-1131, SEM: TSI020302-2010-65), the Madrid Regional Government (SPIR: 50/2010O.23/12/09,TIC2010 and O-PRO: PIE/209/2010) and UPM (Q090935C59) for the support given in the preparation of the paper. The authors thank Synopsys (formerly Optical Research Associates) for granting us the LightTools university license

\section{REFERENCES}

[1] Miñano, J.C., González, J.C. and Benítez, P., "RXI: A high-gain, compact, nonimaging concentrator", Appl. Opt. 34 (34), 7850-7856 (1995)

[2] Muñoz, F., [Sistemas ópticos avanzados de gran compactibilidad con aplicaciones en formación de imagen y en iluminación], Thesis Doctoral, E.T.S.I.Telecomunicación, Madrid (2004)

[3] Muñoz, F., Benítez, P., Dross, O., Miñano, J.C. and Parkyn, B., "Simultaneous multiple surface design of compact air-gap collimators for light-emitting diodes" Opt. Eng. 43 (07), 1522-1530 (2004)

[4] Winston, R., Miñano, J.C. and Benítez, P., [Nonimaging Optics], Elsevier, Academic Press (2004)

[5] Grabovičkić, D., Benítez, P. and Miñano, J.C., " Free-form V-groove reflector design with the SMS method in three dimensions", Opt. Express 19 (S4), A747-A756 (2011)

[6] Benítez, P., Miñano, J.C. and Santamaría, A., "Analysis of microstructured surfaces in two dimensions," Opt. Express 14 (19), 8561-8567 (2006).

[7] Benítez, P., Miñano, J.C., Santamaría, A. and Hernández, M., "On the analysis of rotational symmetric microstructured surfaces", Opt. Express 15 (5), 2219-2233 (2007)

[8] Synopsys software package LightTools, http://www.opticalres.com/ 